\section{Bilateral Extensive Papillomacular Myelination of Retinal Nerve Fibers: A Case Report}

\section{Dear Editor,}

Myelinated retinal nerve fiber (MRNF) layers appear as white or gray-white patches on the surface of the retina with feathery margins correspond to the distribution of ganglion cell axons [1]. It is an uncommon benign developmental anomaly in $0.4 \%$ to $1 \%$ of the population, with a bilateral involvement rate of about 7\% [2]. Patients are mostly asymptomatic, but visual function can be affected depending on the extent, location of the MRNF and coexisting ocular abnormalities. Here, we report a rare case of extensive MRNF involving the papillomacular bundles in both eyes.

A 22-year-old female presented to our clinic with complaints of nystagmus and decreased vision. She had no systemic co-morbidities and family history of ocular disease. Her best-corrected visual acuities were $20 / 100$ in both eyes, and refraction values were $-5.50 /-5.00 \times 175$ in the right eye and $-7.00 /-3.50 \times 10$ in the left eye. The anterior segment was unremarkable, and intraocular pressure were $19 \mathrm{mmHg}$ in the right eye, and $21 \mathrm{mmHg}$ in the left eye. Fundus examination revealed extensive white striated patches consistent with MRNF along the papillomacular bundle in both eyes. Lesions of macular atrophy temporal to fovea were also observed (Fig. 1A, 1B), but there was no peripheral retinal abnormality in both eyes. On fundus autofluorescence, round hypo-autofluorescent lesions with hyper-autofluorescent borders on their superior, inferior, and temporal sides were observed at the region corresponding to the macular atrophic lesion temporal to fovea. In addition, other hypo-autofluorescent lesions nasal to fovea, which were separated from temporal atrophic lesions by normal autofluorescence at the fovea and were partially

Received: May 3, 2021 Final revision: July 16, 2021

Accepted: July 16, 2021
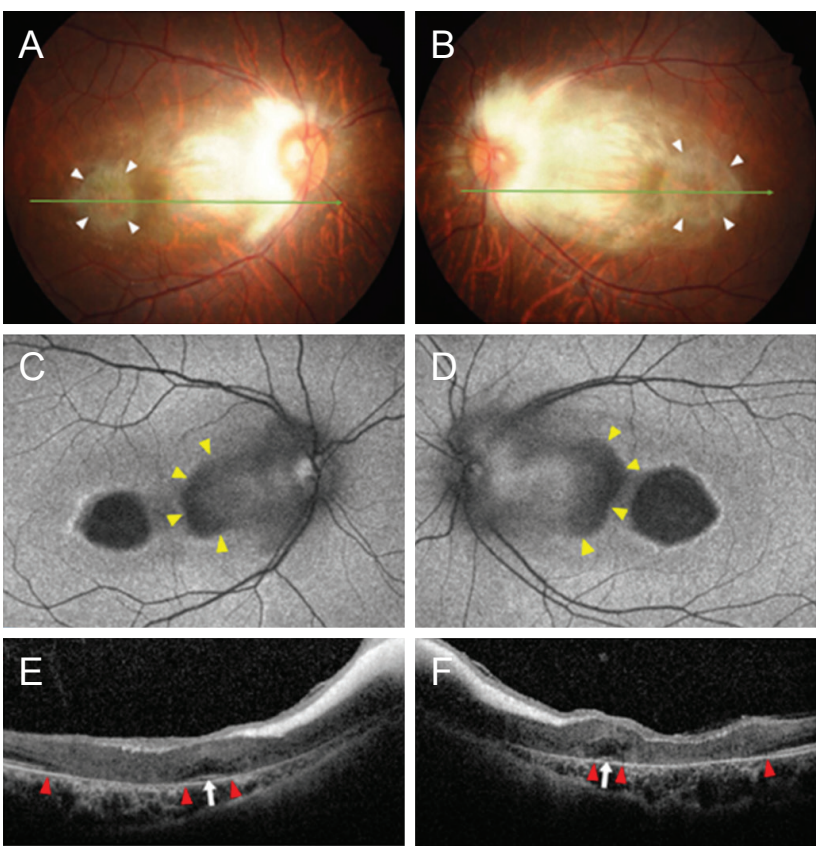

Fig. 1. Images of myelinated retinal nerve fibers (MRNFs) of both eyes of a patient. (A,B) Fundus photographs of both eyes show MRNFs involving the papillomacular bundle extensively. Note the retinal atrophic lesions temporal to the fovea (white arrowheads). (C,D) Fundus autofluorescent images of both eyes show round hypo-autofluorescent lesions at the region corresponding to the retinal atrophic lesion temporal to the fovea. Additional hypo-autofluorescent lesions separated from temporal atrophic lesions by normal autofluorescence at the fovea are observed nasal to the fovea (yellow arrowheads), but they are partially obscured by the MRNF. (E,F) Optical coherence tomography of both eyes shows MRNFs as hyper-reflective layers with tapered edges extending temporal to the fovea. Note the atrophic changes of the outer retina sparing the fovea (white arrows). Boundaries of macula atrophy lesions are indicated with red arrowheads. Informed consent for publication of the clinical images was obtained from the patient.

obscured by the MRNF, were observed (Fig. 1C, 1D). On optical coherence tomography (OCT), MRNF appeared as a hyper-reflective layer with tapered edges extending temporal to fovea. The retinal layer structures of the macula underneath the MRNF were atrophic and replaced by homogenous tissue with moderate reflectivity, except at the fovea where outer nuclear layer and hyper-reflective lines of the ellipsoid zone were preserved (Fig. 1E, 1F). In the electroretinography, scotopic response was normal but photopic response was moderately decreased in both eyes. 
Myelination of optic nerve normally starts at lateral geniculate body and stops posterior to the lamina cribrosa. Oligodendrocytes play an important role in myelination, by proliferation and production of myelin under the influence of hormonal signals during the prenatal period. At lamina cribrosa, the myelination can cease owing to dense astrocytes, which may act as physical barriers. The physiology of MRNF formation has not been fully understood, but it is considered as an abnormal extension of myelination into the retinal nerve fiber layer beyond the lamina cribrosa. It may be due to abnormal migration of oligodendrocytes into the retina either before formation of barrier function of lamina cribrosa or through acquired disruption of lamina cribrosa. Thus, location of the MRNF is usually juxtapapillary and posterior to the equator [3].

The common ocular abnormalities associated with MRNF include anisometropic myopia, amblyopia, and strabismus. In particular, widespread extension of MRNF has been reported to be associated with high myopia [4]. In this case involving extensive papillomacular MRNF in both eyes, high degrees myopia and nystagmus were observed. Although no other coexisting abnormalities were found in this case, a variety of abnormalities, such as craniofacial abnormalities, colobomas, neurofibromatosis, optic nerve hypoplasia have been reported to be associated with MRNFs [2].

Until now, only single report by Ong et al. [5] described bilateral extensive MRNF with papillomacular involvement. In the case, a fundus photograph showed macular atrophic lesions not only temporal but also nasal to fovea, which were obscured by MRNF. This finding is strikingly similar to the finding of the present case, suggesting that extensive MRNFs with papillomacular involvement may be accompanied by macular atrophy, unlike MRNFs of small sizes or those at other retinal locations. Based on spectral domain OCT and fundus autofluorescence, macular atrophy and outer retinal disruption sparing the fovea were confirmed in this case. However, the mechanisms for the atrophic change and foveal preservation are unclear and need to be elucidated.

In conclusion, extensive involvement of bilateral papillomacular bundles by MRNF is a rare condition, and macular atrophy sparing the fovea may be accompanied. Imaging modalities, including fundus autofluorescence and spectral domain OCT, may be helpful to reveal structural changes of the macula obscured by MRNF.

Chae Hyun Song, Un Chul Park

Department of Ophthalmology, Seoul National University

College of Medicine, Seoul, Korea

E-mail(UnChul Park):ucpark@snu.ac.kr

\section{Conflict of Interest}

No potential conflict of interest relevant to this article was reported.

\section{References}

1. Shelton JB, Digre KB, Gilman J, et al. Characteristics of myelinated retinal nerve fiber layer in ophthalmic imaging: findings on autofluorescence, fluorescein angiographic, infrared, optical coherence tomographic, and red-free images. JAMA Ophthalmol 2013;131:107-9.

2. Tarabishy AB, Alexandrou TJ, Traboulsi EI. Syndrome of myelinated retinal nerve fibers, myopia, and amblyopia: a review. Surv Ophthalmol 2007;52:588-96.

3. Rao R, Turkoglu EB, Say E, Shields CL. Clinical features, imaging, and natural history of myelinated retinal nerve fiber layer. Retina 2019;39:1125-32.

4. Straatsma BR, Heckenlively JR, Foos RY, Shahinian JK. Myelinated retinal nerve fibers associated with ipsilateral myopia, amblyopia, and strabismus. Am J Ophthalmol 1979;88(3 Pt 1):506-10.

5. Ong JM, Poornesh KN, El-Hindy N, Goble R. Bilateral macular atrophy and papillomacular myelinated nerve fibre. J Paediatr Child Health 2011;47:67-8. 